\title{
Are Suicidal Thoughts and Behaviors a Temporary Phenomenon in Early Adolescence?
}

\author{
Cornelia Leontine van Vuuren ${ }^{1,2}$ (D), Marcel Franciscus van der Wal ${ }^{1}$, Pim Cuijpers ${ }^{3}$, \\ and Mai Jeanette Maidy Chinapaw²
}

\author{
'Department of Epidemiology, Health Promotion and Healthcare Innovation, Public Health Service (GGD) Amsterdam, The Netherlands \\ 2Department of Public and Occupational Health, Amsterdam Public Health Research Institute, Amsterdam UMC, \\ Vrije Universiteit Amsterdam, The Netherlands \\ ${ }^{3}$ Department of Clinical, Neuro and Developmental Psychology, Amsterdam Public Health Research Institute, \\ Vrije Universiteit Amsterdam, The Netherlands
}

\begin{abstract}
Background: The incidence of first suicidal thoughts and behaviors (STBs) peaks during early adolescence. After experiencing their first STBs, adolescents differ greatly in the extent to which they continue to have STBs. Aim: We determined the course of STBs in Dutch students at two ages: 13-14 years ( $t 1)$ and 15-16 years ( $t 2)$. Methods: Longitudinal data on STBs and sociodemographic factors were collected by self-report $(n=8,499)$. Associations between having STBs at $t 1$ and $t 2$ were determined with multinomial logistic regression analysis. Results: Students who reported suicidal thoughts at baseline $(n=1,077 ; 13 \%)$ reported suicidal thoughts $(O R=6.60 ; 95 \% \mathrm{CI}[5.52,7.88])$ and suicidal attempts $(O R=6.97 ; 95 \% \mathrm{Cl}[4.20,11.54])$ at $t 2$ more often than students with no STBs at $t 1$. Students who reported a suicidal attempt at baseline ( $n=144 ; 2 \%)$ also reported suicidal thoughts and suicidal attempts more often at $t 2(O R=5.98 ; 95 \% \mathrm{Cl}[3.89,9.21] ; O R=30.00 ; 95 \%$ CI [15.84, 56.82], respectively). Limitations: The use of confidential self-reported data and the loss of cases after merging could have biased the results. Conclusion: For a subgroup of adolescents, STBs persisted and worsened over the 2 years. This demonstrates the importance of accurate identification of those at increased risk of suicide, in combination with personalized care.
\end{abstract}

Keywords: suicide, adolescents, suicidal thoughts and behaviors, longitudinal

Recent research showed potential increasing mental health problems and self-harm among adolescents (Morgan et al., 2017; van Vuuren, Uitenbroek, van der Wal, \& Chinapaw, 2018). Early adolescence is also a peak time for the incidence of first suicidal thoughts and behaviors (STBs), such as plans and attempts to die by suicide (Nock et al., 2008). After experiencing these first STBs, adolescents differ greatly in the degree to which they continue to have STBs, from no or decreasing suicidal thoughts to going on to make a suicide plan or attempt (Goldston et al., 2016; Nock et al., 2008). This leaves an important question unanswered: Are STBs generally a temporary phenomenon in early adolescence that naturally fades away, or are preventive interventions needed? Surveys of STBs among adolescents reported mean lifetime estimates of approximately $30 \%$, suggesting that STBs are common in adolescents (Evans, Hawton, Rodham, \& Deeks, 2005; Kokkevi, Rotsika, Arapaki, \& Richardson, 2012). Themes such as life, dying, and suicide are common in thoughts of young adolescents (de Kinder, van Vaerenbergh, \& Vanhoornissen, 2009). In some, STBs may reflect a developing understanding of mortality. In others, STBs may indicate significant risk of a suicidal act (Evans et al., 2005). Evidence suggest that most self-harming behavior in adolescents resolves spontaneously (Moran et al., 2012). However, to the best of our knowledge no other population-based studies have examined the longitudinal course of STBs to test whether the increase in STBs during early adolescence is transient or is a risk factor for continuing STBs. This knowledge is important for accurate identification of suicidal adolescents in need of referral. We therefore determined the course of STBs in Dutch multiethnic students over a period of 2 years.

\section{Compliance With Ethical Standards}

This study is registered at the Dutch Data Protection Authority and meets national ethics and privacy requirements. Medical ethical approval was sought before analysis, but the ethics board ruled that observational studies using anonymized data are not subject to ethical approval. 


\section{Informed Consent}

Passive informed consent was obtained from all individual participants included in the study.

\section{Methods}

All secondary schools in Amsterdam require routine health assessments of their students in the eight and tenth grade by the Amsterdam Public Health Service (GGD). This assessment includes the completion of a self-reported electronic health questionnaire, during school hours under supervision of a teacher and nurse. The data in this study were obtained from this questionnaire.

Suicidal thoughts and suicide attempts were assessed by two questions: (1) "During the past 12 months, have you ever seriously thought about ending your life?"; and (2) "During the past 12 months, have you made an attempt to end your life?" - in line with widely accepted definitions of suicidal thoughts and attempts (Silverman, Berman, Sanddal, O'Carroll, \& Joiner, 2007a, 2007b). The response categories for the first question were dichotomized into no (never) or yes (other categories). The response categories for the second question were no or yes. Sociodemographic factors included age, sex, ethnic origin, educational level, and family composition. Details about this research project, including ethical procedures, are described elsewhere (van Vuuren et al., 2018).

To explore the 2-year longitudinal associations, we used data from three cohorts. The first data wave was collected in school years 2010-2011 (Cohort 1), 2011-2012 (Cohort 2), and 2012-2013 (Cohort 3). At that time the students were in the eighth grade, the second year of Dutch secondary education and were 13-14 years old $(t 1)$. The second wave ( $t 2)$ was collected 2 years later. Data files at $t 1$ and $t 2$ were merged based on birth date, school, and postal code.

Multinomial logistic regression analyses were used to assess associations between having STBs at $t 1$ and $t 2$, adjusting for the aforementioned sociodemographic factors at $t 1$. The predictor variable was Having STBs at $t 1$ with the categories "no suicidal thoughts and attempts" (reference category), "suicidal thoughts, without attempts," and "suicidal attempts." The outcome variable was Having STBs at $t 2$, with the same three categories. The multinomial analysis compared "suicidal thoughts" versus "no suicidal thoughts and attempts," and "suicidal attempts" versus "no suicidal thoughts and attempts."

We performed an additional descriptive analysis, using chi-squared tests, to examine whether those who continued to report STBs in follow-up differed from those who reported STBs at $t 1$ only, in terms of sociodemographic characteristics, psychosocial problems, unsafe environment, and substance use reported at $t 1$. All variables were identified as suicide risk factors and warning signs in the meta-analysis of Franklin et al. (2017).

\section{Results}

At both $t 1(n=15,518)$ and $t 2(n=14,805)$ the response rates were approximately $90 \%$. Data at $t 1$ and $t 2$ could be merged for 8,499 students. This merged sample was comparable with the initial sample $(t 1)$, based on sex, ethnic origin, educational level, and family composition.

At $t 1,1,077$ students (12.7\%) reported suicidal thoughts and 144 students $(1.7 \%)$ reported attempted suicide, while 7,278 (85.6\%) students did not report suicidal thoughts or attempts. Of the 1,221 students with STBs at $t 1,363$ students $(29.7 \%)$ reported STBs at $t 2$. Relative to their peers without STBs at $t 1$, students with su-

Table 1. Longitudinal associations between having STBs at age 14 ( $t 1)$ and having STBs 2 years later ( $t 2)$

\begin{tabular}{|c|c|c|c|c|c|}
\hline$N=8,499$ & $\%$ & OR Crude & $95 \% \mathrm{Cl}$ & OR Adjusted $\dagger$ & $95 \% \mathrm{Cl}$ \\
\hline \multicolumn{6}{|l|}{ Suicidal thoughts ${ }^{a}(t 2)$} \\
\hline No STBs $(t 1)$ & 4.8 & 1 & & 1 & \\
\hline Suicidal thoughts ${ }^{a}(t 1)$ & 26.3 & $7.33^{*}$ & {$[6.16,8.72]$} & $6.60 *$ & {$[5.52,7.88]$} \\
\hline Suicide attempts $(t 1)$ & 21.5 & $6.45^{\star}$ & {$[4.24,9.81]$} & $5.98 *$ & {$[3.89,9.21]$} \\
\hline \multicolumn{6}{|l|}{ Suicide attempts (t2) } \\
\hline No STBs $(t 1)$ & 0.5 & 1 & & 1 & \\
\hline Suicidal thoughts ${ }^{a}(t 1)$ & 2.9 & $8.50 *$ & {$[5.17,13.94]$} & $6.97 *$ & {$[4.20,11.54]$} \\
\hline Suicide attempts $(t 1)$ & 12.5 & $39.59 *$ & {$[21.54,72.79]$} & $30.0 *$ & {$[15.84,56.82]$} \\
\hline
\end{tabular}

Note. $O R=$ Odds Ratio; $95 \% \mathrm{Cl}=95 \%$ confidence interval. STBs = suicidal thoughts and behaviors. ${ }^{2}$ Without attempts. $\dagger$ Adjusted for sociodemographic factors at $t 1$.

$\star p<.001$ 
icidal thoughts at $t 1$ reported suicidal thoughts at $t 2$ five times $(26.3 \%$ vs. $4.8 \%)$ more often $(O R=6.60 ; 95 \% \mathrm{CI}$ $[5.52,7.88])$ and suicidal attempts at $t 2$ six times $(2.9 \%$ vs. $0.5 \%)$ more often $(O R=6.97 ; 95 \%$ CI $[4.20,11.54])$. Students reporting a suicidal attempt at $t 1$ reported suicidal thoughts four times $(21.5 \%$ vs. $4.8 \%)$ more often $(O R=5.98 ; 95 \% \mathrm{CI}[3.89,9.21])$ and suicidal attempts 25 times more often $(12.5 \%$ vs. $0.5 \%, O R=30.00 ; 95 \%$ CI $[15.84,56.82])$ than their peers without STBs at $t 1$ (Table 1$)$.

Students who persisted having STBs at $t 2$, were more often girls, had a high level of education, were more often not living with both parents, and reported psychosocial problems, physical abuse, or mental abuse more often at $t 1$, compared with the group who reported STBs at $t 1$ only. See Table 1 in Electronic Supplementary Material 1.

\section{Discussion}

Our results indicate that STBs among young adolescents are quite common, but that for most adolescents STBs disappear within 2 years. However, our study also shows that a subgroup of adolescents sustain STBs for a longer period of time. Similar to previous studies (Franklin et al., 2017), we found that these adolescents reported psychosocial problems more often and grew up in an unsafe environment. This subgroup may be at increased risk for suicide.

Strengths of our study include the longitudinal, population-based design and the high participation rate. Possible limitations are the use of confidential instead of anonymous data, that the surveys were only 2 years apart, which might have increased the observed levels of continuity, and the loss of cases after merging the data. However, this loss was mainly due to changing schools, or a missing postal code or birth date. Moreover, the merged sample was comparable with the initial sample based on sex, ethnic origin, educational level, and family composition. We therefore assume that the merged sample was broadly representative for 13-14-years-olds in Amsterdam. Another limitation is that self-reported suicide attempts have limited validity owing to possible incorrect interpretation of the questions by respondents (Silverman et al., 2007a, 2007b).

Our findings emphasize the importance for practitioners to identify adolescents who develop worrisome STBs. Unfortunately, there is no evidence that risk stratification contributes to suicide prevention (Large, Ryan, Carter, \& Kapur, 2017). Existing risk factors or a combination of risk factors appeared weak and inaccurate predictors of STBs. A possible solution to improve prediction of STBs is to include the different degrees of suicidality in screening methods. Screenings methods using machine learning-based risk algorithms could improve prediction and screening (Franklin et al., 2017). Nevertheless, care based on adolescents' current treatment needs for a wide variety of treatable problems that go with STBs (e.g., depression, substance misuse, school problems) remains very important (Large et al., 2017).

\section{Electronic Supplementary Material}

The electronic supplementary material is available with the online version of the article at https://doi.org/ 10.1027/0227-5910/a000680

ESM 1. Percentages of sociodemographic characteristics, psychosocial problems, unsafe environment, and substance use of students reporting suicidal thoughts or attempts (STBs) at $t 1$ only, and of students who persisted having STBs at $t 2$

\section{References}

Evans, E., Hawton, K., Rodham, K., \& Deeks,J.(2005). The prevalence of suicidal phenomena in adolescents: A systematic review of population-based studies. Suicide and Life-Threatening Behavior, 35(3), 239-250. https://doi.org/10.1521/suli.2005.35.3.239

Franklin, J. C., Ribeiro, J. D., Fox, K. R., Bentley, K. H., Kleiman, E. M., Huang, X., ... Nock, M. K. (2017). Risk factors for suicidal thoughts and behaviors: A meta-analysis of 50 years of research. Psychological Bulletin, 143(2), 187-232. https://doi. org/10.1037/bul0000084

Goldston, D. B., Erkanli, A., Daniel, S. S., Heilbron, N., Weller, B. E., \& Doyle, O. (2016). Developmental trajectories of suicidal thoughts and behaviors from adolescence through adulthood. Journal of the American Academy of Child \& Adolescent Psychiatry, 55(5), 400-407, e401. https://doi.org/10.1016/j.jaac.2016.02.010

Kinder de, G., Vaerenbergh van, G., \& Vanhoornissen, T. (2009). Psychologische vragen die leven bij basisschoolleerlingen tussen tien en twaalf jaar [Psychological questions that live with primary school pupils between ten and twelve years old]. Kind en Adolescent (Child and Adolescent), 30(2), 82-94. https://doi. org/10.1007/BF03087938

Kokkevi, A., Rotsika, V., Arapaki, A., \& Richardson, C. (2012). Adolescents' self-reported suicide attempts, self-harm thoughts and their correlates across 17 European countries. Journal of Child Psychology and Psychiatry, 53(4), 381-389. https://doi. org/10.1111/j.1469-7610.2011.02457.x

Large, M. M., Ryan, C. J., Carter, G., \& Kapur, N. (2017). Can we usefully stratify patients according to suicide risk? BMJ, 359, j4627. https://doi.org/10.1136/bmj.j4627

Moran, P., Coffey, C., Romaniuk, H., Olsson, C., Borschmann, R., Carlin, J. B., \& Patton, G. C. (2012). The natural history of self-harm from adolescence to young adulthood: A population-based cohort study. Lancet, 379(9812), 236-243. https://doi.org/10.1016/ S0140-6736(11)61141-0 
Morgan, C., Webb, R. T., Carr, M. J., Kontopantelis, E., Green, J., Chew-Graham, C. A., ... Ashcroft, D. M. (2017). Incidence, clinical management, and mortality risk following self harm among children and adolescents: Cohort study in primary care. BMJ, 359, j4351. https://doi.org/10.1136/bmj.j4351

Nock, M. K., Borges, G., Bromet, E.J., Cha, C. B., Kessler, R. C., \& Lee, S. (2008). Suicide and suicidal behavior. Epidemiologic Reviews, 30,133-154. https://doi.org/10.1093/epirev/mxn002

Silverman, M. M., Berman, A. L., Sanddal, N. D., O'Carroll, P.W., \& Joiner, T. E. (2007a). Rebuilding the tower of Babel:A revised nomenclature for the study of suicide and suicidal behaviors. Part 1: Background, rationale, and methodology. Suicide and Life-Threatening Behavior, 37(3), 248-263. https://doi.org/10.1521/suli.2007.37.3.248

Silverman, M. M., Berman, A. L., Sanddal, N. D., O'Carroll, P. W., \& Joiner, T. E. (2007b). Rebuilding the tower of Babel: A revised nomenclature for the study of suicide and suicidal behaviors. Part 2: Suicide-related ideations, communications, and behaviors. Suicide and Life-Threatening Behavior, 37(3), 264-277. https:// doi.org/10.1521/suli.2007.37.3.264

van Vuuren, C. L., Uitenbroek, D. G., van der Wal, M. F., \& Chinapaw, M. J. M. (2018). Sociodemographic differences in 10-year time trends of emotional and behavioural problems among adolescents attending secondary schools in Amsterdam, The Netherlands. European Child \& Adolescent Psychiatry, 27(12), 16211631. https://doi.org/10.1007/s00787-018-1157-5

\section{History}

Received June 13, 2019

Revision received November 26, 2019

Accepted November 30, 2019

Published online March 31, 2020

\section{Conflict of Interest}

The authors declare that that they have no conflict of interest.

\section{Authorship}

C.L. van Vuuren: conception and design of the work, data collection, analysis and interpretation, drafting the article, and final approval of the version to be published.

M.F. van der Wal, P. Cuijpers, M.J.M. Chinapaw: data interpretation, critical revision of the article, and final approval of the version to be published.

\section{ORCID}

Cornelia van Vuuren

(iD) https://orcid.org/0000-0002-9780-769X

\section{Cornelia Leontine van Vuuren}

Public Health Service (GGD) Amsterdam

Nieuwe Achtergracht 100

1018 WT Amsterdam

The Netherlands

lvvuuren@ggd.amsterdam.nl

Leonie van Vuuren is a PHD student at the Amsterdam University Medical Centers, The Netherlands, and researcher at the Public Health Service Amsterdam. In recent years she worked as project director at the Youth Health Monitor. Her research focuses on the epidemiology and longitudinal trajectories of suicidal thoughts and behaviors among adolescents.

Marcel van der Wal, PhD, is an epidemiologist and Head of Youth at the Department of Epidemiology, Health Promotion and Health Care Innovation, Public Health Service Amsterdam, The Netherlands. His team conducts scientific research into effectiveness of prevention programs and continuously examines the health and lifestyle of Amsterdam youth.

Pim Cuijpers is Professor of Clinical Psychology at the Vrije Universiteit Amsterdam, The Netherlands, and Head of the Department of Clinical, Neuro and Developmental Psychology. He is also director of the WHO Collaborating Centre for Research and Dissemination of Psychological Interventions in Amsterdam. He has authored almost 900 publications.

Mai Chinapaw is University Research Professor at the Amsterdam University Medical Centers, The Netherlands, chair of the section Child Health \& Care Research and director of the research program Health Behaviours and Chronic Diseases of the Amsterdam Public Health research institute. She has published more than 270 papers in peer-reviewed journals. 